\title{
On the Construction of Spherical Designs
}

L.C. LEAL JUNIOR ${ }^{1}$, V.A. MENEGATTO ${ }^{2}$, Departamento de Matemática, ICMCUSP - São Carlos, Cx.P. 668, 13560-970 São Carlos SP, Brasil.

\begin{abstract}
We study special subsets of the unit sphere in $\mathbb{R}^{m}, m \geq 2$, the socalled spherical designs in the literature. Among other things we introduce a new equivalence for the concept and investigate the construction of designs through rotations of $\mathbb{R}^{m}$ and projections over the equator of the sphere.
\end{abstract}

\section{Introduction}

The standard definition of spherical design requires that certain equally weighted cubature formulas be exact for spherical polynomials up to a certain degree. Let us be a little bit more specific. If $S^{m-1}$ denotes the unit sphere in $\mathbb{R}^{m}$ and $q$ is a positive integer, a finite nonempty subset $W$ of $S^{m-1}$ is said to be a $q$-design if

$$
\frac{1}{\sigma_{m-1}} \int_{S^{m-1}} f(x) d \sigma(x)=\frac{1}{|W|} \sum_{x \in W} f(x), \quad f \in \mathcal{P}_{q} .
$$

Here, $\sigma_{m-1}$ stands for the surface area of $S^{m-1}$, the integration is the usual one over $S^{m-1},|W|$ is the cardinality of $W$ and $\mathcal{P}_{q}$ is the set of all polynomials in $m$ variables having degree at most $q$. The concept has applications in code theory, discrepancy and combinatorics, approximation theory, Monte-Carlo methods, etc. We refer the reader to many old references authored by Goethals, Delsarte and Seidel where the concept was investigated quite well.

Since the abstract of this paper describes what the aim of the paper is, we complete the section presenting the basic material needed in the forthcoming sections. The proofs will be omitted since most of them are well known. The basic references are $[2,7,9,10]$.

We shall write $\mathcal{H}_{q}$ to denote the subset of $\mathcal{P}_{q}$ formed by its homogeneous elements and $\mathcal{A}_{q}$ to denote the subset of $\mathcal{H}_{q}$ formed by harmonic polynomials. Throughout the paper, we shall be dealing with the elements of these sets restrict to $S^{m-1}$. The notation for the resulting restricted spaces will be the same. Usually, the elements of $\mathcal{H}_{q}$ restricted to $S^{m-1}$ are called $m$-dimensional spherical harmonics of degree $q$.

The basic metric structure used in the paper is that of $L^{2}\left(S^{m-1}\right)$ as explained in the references quoted above. As so, the sets $\mathcal{A}_{q}$ become orthogonal subspaces of

\footnotetext{
${ }^{1}$ jhcleal@gmail.com; partially supported by CAPES.

${ }^{2}$ menegatt@icmc.usp.br; partially supported by CNPq Grant\#470820/2004-7
} 
$L^{2}\left(S^{m-1}\right)$ for different values of $q$. Every element $p$ of $\mathcal{H}_{q}$ has a decomposition in the form

$$
p=\sum_{j=0}^{\lfloor q / 2\rfloor} h_{q-2 j}, \quad h_{q-2 j} \in \mathcal{A}_{q-2 j}, \quad j=0,1, \ldots,\lfloor q / 2\rfloor .
$$

In some passages of the text we shall deal with the Legendre polynomial $R_{q}^{m}$ of degree $q$ associated with the integer $m$, as described in [7]. It is normalized by the relation $R_{q}^{m}(1)=1$. If $m \geq 3$, it relates to the Gegenbauer polynomial $P_{q}^{(m-2) / 2}$ of degree $q$ associated with the dimension $(m-2) / 2$ as follows ([7, 11]):

$$
R_{q}^{m}=\frac{(m-3) ! q !}{(q+m-3) !} P_{q}^{(m-2) / 2} .
$$

The orthogonality of these polynomials refers to the inner product

$$
\langle f, g\rangle^{\prime}:=\int_{-1}^{1} f(t) g(t)\left(1-t^{2}\right)^{(m-3) / 2} d t .
$$

The following formula, known as the addition formula, makes the bridge between spherical harmonics and Legendre polynomials:

$$
\sum_{j=1}^{N_{q}} Y_{j}^{q}(x) Y_{j}^{q}(y)=\frac{N_{q}}{\sigma_{m-1}} R_{q}^{m}(x \cdot y), \quad x, y \in S^{m-1} .
$$

Here, $\left\{Y_{j}^{q}: j=1,2, \ldots, N_{q}^{m}\right\}$ is a fixed orthonormal basis of $\mathcal{A}_{q}$ and $\cdot$ is the usual inner product of $\mathbb{R}^{m}$. An induction process shows that it is possible to find a fundamental system for $\mathcal{A}_{q}$, that is, a subset $\left\{x_{1}, x_{2}, \ldots, x_{N_{q}^{m}}\right\}$ of $S^{m-1}$ for which the matrix $\left(f_{j}\left(x_{i}\right)\right)$ is invertible for every basis $\left\{f_{1}, f_{2}, \ldots, f_{N_{q}^{m}}^{q}\right\}$ of $\mathcal{A}_{q}$.

The Funk-Hecke formula states that

$$
\int_{S^{m-1}} K(x \cdot y) h(y) d \sigma(y)=\alpha_{q}(K) h(x), \quad x \in S^{m-1},
$$

whenever $h \in \mathcal{A}_{q}, K$ is a function with domain $[-1,1]$ and the integral makes sense. The coefficient $\alpha_{q}(K)$ is calculated through the formula

$$
\alpha_{q}(K):=\sigma_{m-2} \int_{-1}^{1} K(t) R_{q}^{m}(t)\left(1-t^{2}\right)^{(m-3) / 2} d t .
$$

An immediate consequence of the formula is the reproducing property

$$
h(x)=\frac{N_{q}}{\sigma_{m-1}} \int_{S^{m-1}} R_{q}^{m}(x \cdot y) h(y) d \sigma(y), \quad x \in S^{m-1}, \quad h \in \mathcal{A}_{q} .
$$

Not so popular is the addition formula for Gegenbauer polynomials ([1]). For real numbers $\theta, \phi$ and $\vartheta$, it reads

$$
\begin{aligned}
P_{q}^{(m-2) / 2}(\cos \theta \cos \phi+\sin \theta \operatorname{sen} \phi \cos \vartheta) & =P_{q}^{(m-2) / 2}(\cos \theta) P_{q}^{(m-2) / 2}(\cos \phi) \\
& +\sum_{i=1}^{q} \beta_{i, q} Q_{i}(\theta) Q_{i}(\phi) P_{i}^{(m-3) / 2}(\cos \vartheta)
\end{aligned}
$$


where $\beta_{i, q}>0, i=1,2, \ldots, q$ and

$$
Q_{i}(\theta)=\operatorname{sen}^{i} \theta P_{q-i}^{(m-2+2 i) / 2}(\cos \theta), \quad i=1,2, \ldots, q, \quad \theta \in \mathbb{R} .
$$

Finally, we recall Dougall's linearization formula $[1,3,8]$ : If $k$ and $n$ are nonnegative integers, then

$$
R_{k}^{m} R_{n}^{m}=\sum_{j=0}^{k \wedge n} \alpha_{j}^{k, n} R_{k+n-2 j}^{m}
$$

where every coefficient $\alpha_{j}^{k, n}$ is positive.

\section{Equivalences for the Concept of Design}

The concept of design has many equivalent formulations. The definitions of design on its own implies the following facts: a nonempty subset $W$ of $S^{m-1}$ is a 1-design if and only if $\sum_{x \in W} x=0$. If $W \subset S^{m-1}$ is a $q$-design and $q_{1} \in\{1,2, \ldots, q\}$ then $W$ is a $q_{1}$-design. Below, we recall the most recent equivalence for the concept, due to Yudin ([12]). It allows us to present nontrivial examples of designs in an elementary way.

Theorem 2.1. Let $W$ be a finite nonempty subset of $S^{m-1}$. The following assertions are equivalent:

(i) $W$ is a q-design;

(ii) $\sum_{x \in W} R_{k}^{m}(x \cdot y)=0, y \in S^{m-1}, k=1,2, \ldots, q$;

(iii) It holds

$$
\frac{1}{|W|} \sum_{x \in W}(x \cdot y)^{k}=\alpha_{k}, \quad y \in S^{m-1}, \quad k=1,2, \ldots, q,
$$

where

$$
\alpha_{k}= \begin{cases}0, & k \text { odd } \\ \frac{\sigma_{m-2}}{\sigma_{m-1}} \int_{-1}^{1} t^{k}\left(1-t^{2}\right)^{(m-3) / 2} d t, & k \text { even } .\end{cases}
$$

The Funk-Hecke formula shows that the integral appearing in the previous formula can be re-written as

$$
\frac{1}{\sigma_{m-1}} \int_{S^{m-1}}(y \cdot x)^{k} d \sigma(x),
$$

where $y$ is an arbitrary point of $S^{m-1}$.

The set $W_{1}=\{(1,0),(0,1),(-1,0),(0,-1)\}$ is a 3-design in $S^{1}$. Indeed, we verify that $W_{1}$ satisfies Condition (iii) above. Let $y=\left(y_{1}, y_{2}\right) \in S^{1}$. If $k=1$, the sum in (iii) takes the form $y_{1}-y_{1}+y_{2}-y_{2}=0$. If $k=2$, it reduces itself to $2\left(y_{1}^{2}+y_{2}^{2}\right):=2\|y\|^{2}$. On the other hand, taking $y=(1,0)$ in $(2.3)$ and using polar coordinates we obtain

$$
\alpha_{2}=\frac{1}{2 \pi} \int_{S^{1}}\left((1,0) \cdot\left(y_{1}, y_{2}\right)\right)^{2} d \sigma(y)=\frac{1}{2 \pi} \int_{0}^{2 \pi} \cos ^{2} t d t=\frac{1}{2} .
$$


Finally, if $k=3, y_{1}^{3}+\left(-y_{1}\right)^{3}+y_{2}^{3}+\left(-y_{2}\right)^{3}=0$. From Theorem 2.1, the desired conclusion follows.

The set $W_{2}=\{(1,0,0),(0,1,0),(0,0,1),(-1,0,0),(0,-1,0),(0,0,-1)\}$ is a 3 design in $S^{2}$. To see that we verify that $W_{2}$ satisfies Condition (ii) in Theorem 2.1. The low degree Legendre polynomials are given by $R_{1}^{3}(t)=t, 2 R_{2}^{3}(t)=3 t^{2}-1$ and $2 R_{3}^{3}(t)=5 t^{3}-3 t$. Fixing $y=\left(y_{1}, y_{2}, y_{3}\right) \in S^{2}$ we have the following possibilities: if $k=1$,

$$
\sum_{x \in W} R_{1}^{3}(x \cdot y)=y_{1}+y_{2}+y_{3}-y_{1}-y_{2}-y_{3}=0
$$

if $k=2$,

$$
\sum_{x \in W} R_{2}^{3}(x \cdot y)=\frac{1}{6}\left(6 y_{1}^{2}+6 y_{2}^{2}+6 y_{3}^{2}-6\right)=3\|y\|^{2}-3=0 ;
$$

Finally, if $k=3$,

$$
\sum_{x \in W} R_{3}^{3}(x \cdot y)=\frac{1}{2}\left(5 y_{1}^{3}+5 y_{2}^{3}+5 y_{3}^{3}-5 y_{1}^{3}-5 y_{2}^{3}-5 y_{2}^{3}-3 y_{1}-3 y_{2}-3 y_{3}+3 y_{1}+3 y_{2}+3 y_{3}\right)=0 .
$$

Another criteria for a set $W$ to be a $q$-design are given below. We include a proof here just because we were unable to find one in the literature. Arguments similar to the ones used in the proof can be found in $[4,5,12]$. We write $\mathcal{O}_{m}$ to denote the set of all orthogonal transformations on $\mathbb{R}^{m}$.

Theorem 2.2. Let $W$ be a finite nonempty subset of $S^{m-1}$. The following assertions are equivalent:

(i) $W$ is a q-design;

(ii) It holds

$$
\frac{1}{|W|} \sum_{x \in W} p(\rho(x))=\frac{1}{\sigma_{m-1}} \int_{S^{m-1}} p(x) d \sigma(x), p \in \mathcal{H}_{k}, k=0,1, \ldots, q, \rho \in \mathcal{O}_{m} .
$$

(iii) $\sum_{x \in W} h(x)=0, h \in \mathcal{A}_{k}, k=1,2, \ldots, q$;

(iv) $\sum_{x \in W} p(\rho(x))=\sum_{x \in W} p(x), p \in \mathcal{H}_{k}, k=0,1, \ldots, q, \rho \in \mathcal{O}_{m}$.

Proof. Let $k \in\{0,1, \ldots, q\}, p \in \mathcal{H}_{k}$ and $\rho \in \mathcal{O}_{m}$. If $(i)$ holds, the invariance of the Lebesgue measure with respect of elements of $\mathcal{O}_{m}$ on $S^{m-1}$ and the fact that $p \circ \rho \in \mathcal{P}_{q}$ justify the equalities

$$
\sum_{x \in W} p(\rho(x))=\frac{1}{\sigma_{m-1}} \int_{S^{m-1}} p(x) d \sigma\left(\rho^{*}(x)\right)=\frac{1}{\sigma_{m-1}} \int_{S^{m-1}} p(x) d \sigma(x),
$$

where $\rho^{*}$ is the adjoint of $\rho$. Thus, (ii) follows. To verify that the later implies (iii), it suffices to take $\rho$ as the identity transformation and to observe that $\mathcal{A}_{k} \subset \mathcal{H}_{k}$. Next, assume (iii) holds. Let $k \in\{1,2, \ldots, q\}, p \in \mathcal{H}_{k}$ e $\rho \in \mathcal{O}_{m}$. Decomposing $p$ according to (1.2), we shall establish (iv) in two steps. If $k$ is odd, then $k-2 j \geq 1$, $j=0,1, \ldots,\lfloor k / 2\rfloor$ and, consequently,

$$
\sum_{x \in W} p(x)=\sum_{j=0}^{\lfloor k / 2\rfloor} \sum_{x \in W} h_{k-2 j}(x)=0 .
$$


Since $h_{k-2 j} \circ \rho \in \mathcal{A}_{k}, j=0,1, \ldots,\lfloor k / 2\rfloor$, a similar procedure leads to the formula $\sum_{x \in W} p(\rho(x))=0$. If $k$ is even, we proceed in the same way to obtain

$$
\sum_{x \in W} p(x)=|W|=\sum_{x \in W} p(\rho(x)) .
$$

In both cases, we are led to the equality in $(i v)$. To finish the proof, we show that (iv) implies (iii) in Theorem 2.1. Let $k \in\{0,1, \ldots, q\}$. For every $y \in S^{m-1}$, let $\rho_{y} \in \mathcal{O}_{m}$ be chosen so that the coordinates of $y$ with respect to the canonical basis of $\mathbb{R}^{m}$ be, respectively, the entries in the first row in the matrix representation of $\rho_{y}$ with respect to that same basis. Then,

$$
\sum_{x \in W}(x \cdot y)^{k}=\sum_{x \in W} p\left(\rho_{y}(x)\right)
$$

where $p(x)=x_{1}^{k}\left(x_{1}=\right.$ the first component of $\left.x\right)$. If $(i v)$ holds, we conclude that

$$
\sum_{x \in W}(x \cdot y)^{k}=\sum_{x \in W} p(x)
$$

Thus, the function $y \in S^{m-1} \rightarrow \sum_{x \in W}(x \cdot y)^{k}$ is a constant $\beta$, not depending on $y$. However, it is easily seen that

$$
\beta=\frac{1}{\sigma_{m-1}} \int_{S^{m-1}} \beta d \sigma(y)=\sum_{x \in W} \frac{1}{\sigma_{m-1}} \int_{S^{m-1}}(x \cdot y)^{k} d \sigma(y) .
$$

It is now clear that $\beta=|W| \alpha_{k}$, where $\alpha_{k}$ is given in (2.2).

\section{The Results}

The following nice equivalence criteria for the concept of design is a consequence of the results in the previous section. As far as we know, it has not been published yet.

Theorem 3.1. Let $W$ be a finite nonempty subset of $S^{m-1}$. The following assertions are equivalent:

(i) $W$ is a q-design;

(ii) $\sum_{x \in W} R_{k}^{m}(\rho(x) \cdot y)=\sum_{x \in W} R_{k}^{m}(x \cdot y), k=1,2, \ldots, q, y \in S^{m-1}, \rho \in \mathcal{O}_{m}$.

Proof. Since every function $x \in S^{m-1} \rightarrow R_{k}^{m}(x \cdot y), y \in S^{m-1}$ is an element of $\mathcal{H}_{k}$, one implication follows directly from the previous theorem. As for the other, it suffices to verify that $(i i)$ implies Condition $(i v)$ in Theorem 2.2. To do that, in view of (1.2), it suffices to verify that condition in the case where $h \in \mathcal{A}_{k}, k=0,1, \ldots, q$. The case $k=0$ is trivial. As for the others, fix $k \in\{1,2, \ldots, q\}$ and let $h \in \mathcal{A}_{k}$. Observing that $\alpha_{k}\left(R_{k}^{m}\right)>0$, the Funk-Hecke formula leads to

$$
\begin{aligned}
\sum_{x \in W} h(x) & =\frac{1}{\alpha_{k}\left(R_{k}^{m}\right)} \sum_{x \in W} \int_{S^{m-1}} R_{k}^{m}(x \cdot y) h(y) d \sigma(y) \\
& =\frac{1}{\alpha_{k}\left(R_{k}^{m}\right)} \int_{S^{m-1}}\left(\sum_{x \in W} R_{k}^{m}(x \cdot y)\right) h(y) d \sigma(y) .
\end{aligned}
$$


If (ii) holds, the equality

$$
\sum_{x \in W} h(x)=\sum_{x \in W} h(\rho(x)), \quad \rho \in \mathcal{O}_{m},
$$

follows from the previous formula.

To proceed, we assume that $m \geq 3$ and introduce additional notation. Making use of a fixed pole $\varepsilon$ on $S^{m-1}$, we shall decompose a point $x \in S^{m-1}$ in the following form

$$
x=\lambda \varepsilon+\sqrt{1-\lambda^{2}} x_{\varepsilon}^{\prime}, \quad \lambda \in[-1,1], \quad x_{\varepsilon}^{\prime} \in S_{\varepsilon}^{m-1},
$$

where $S_{\varepsilon}^{m-1}$ is the equator of $S^{m-1}$, orthogonal to $\varepsilon$. The set $S_{\varepsilon}^{m-1}$ is in fact a copy of $S^{m-2}$ isometrically embedded in $S^{m-1}$. The embedding itself will be denoted by $\Psi_{\varepsilon}$.

Given a finite nonempty subset $W$ of $S^{m-1}, W_{\varepsilon}^{\prime}$ will denote the spherical projection of $W$ from $\varepsilon$ onto the equator of $S^{m-1}$, that is, $W_{\varepsilon}^{\prime}:=\left\{x_{\varepsilon}^{\prime}: x \in W\right\}$. In particular, $S_{\varepsilon}^{m-1}=\left\{x_{\varepsilon}^{\prime}: x \in S^{m-1}\right\}$.

Theorem 3.2 below provides a concise method to construct spherical designs in $S^{m-2}$ from spherical designs in $S^{m-1}$.

Theorem 3.2. Let $W$ be a finite nonempty subset of $S^{m-1}$ and $\varepsilon \in S^{m-1}$. Assume there exists $\lambda \in(-1,1) \backslash \cup_{k=0}^{q-1}\left\{t: R_{q-k}^{m+k}(t)=0\right\}$ so that $W \subset\left\{x \in S^{m-1}: x \cdot \varepsilon=\lambda\right\}$. If $W$ is a q-design in $S^{m-1}$ then $\Psi_{\varepsilon}^{-1}\left(W_{\varepsilon}^{\prime}\right)$ is a q-design in $S^{m-2}$.

Proof. We intend to use Theorem 2.1. Using (3.2), it is easily seen that

$$
\sum_{x \in W} \sum_{y \in W} R_{k}^{m}(x \cdot y)=\sum_{x \in W} \sum_{y \in W} R_{k}^{m}\left(\lambda^{2}+\left(1-\lambda^{2}\right) x_{\varepsilon}^{\prime} \cdot y_{\varepsilon}^{\prime}\right), \quad x, y \in S^{m-1} .
$$

Since the map $x \in W \rightarrow x_{\varepsilon}^{\prime} \in W_{\varepsilon}^{\prime}$ is one-to-one, it follows that

$$
\sum_{x \in W} \sum_{y \in W} R_{k}^{m}\left(\lambda^{2}+\left(1-\lambda^{2}\right) x_{\varepsilon}^{\prime} \cdot y_{\varepsilon}^{\prime}\right)=\sum_{x_{\varepsilon}^{\prime} \in W_{\varepsilon}^{\prime}} \sum_{y_{\varepsilon}^{\prime} \in W_{\varepsilon}^{\prime}} R_{k}^{m}\left(\lambda^{2}+\left(1-\lambda^{2}\right) x_{\varepsilon}^{\prime} \cdot y_{\varepsilon}^{\prime}\right) .
$$

If $S_{k}$ denotes the very last sum in (3.4), we can use (1.3) and the addition formula for Gegenbauer polynomials to obtain

$$
\begin{aligned}
S_{k} & =\sum_{x_{\varepsilon}^{\prime} \in W_{\varepsilon}^{\prime}} \sum_{y_{\varepsilon}^{\prime} \in W_{\varepsilon}^{\prime}} \sum_{l=0}^{k} \beta_{l, k}^{m}\left(1-\lambda^{2}\right)^{l}\left(R_{k-l}^{m+l}(\lambda)\right)^{2} R_{l}^{m-1}\left(x_{\varepsilon}^{\prime} \cdot y_{\varepsilon}^{\prime}\right) \\
& =\sum_{l=0}^{k} \beta_{l, k}^{m}\left(1-\lambda^{2}\right)^{l}\left(R_{k-l}^{m+l}(\lambda)\right)^{2} \sum_{x_{\varepsilon}^{\prime} \in W_{\varepsilon}^{\prime}} \sum_{y_{\varepsilon}^{\prime} \in W_{\varepsilon}^{\prime}} R_{l}^{m-1}\left(x_{\varepsilon}^{\prime} \cdot y_{\varepsilon}^{\prime}\right),
\end{aligned}
$$

where

$$
\beta_{l, k}^{m}=\frac{(m-3) ! k !}{(k+m-3) !} \beta_{l, k}, \quad l=0,1, \ldots, k .
$$


If $W$ is a $q$-design, (3.3) and Theorem 2.1 imply that $S_{k}=0, k=1,2, \ldots, q$. The quadratic forms appearing in the expression that calculates $S_{k}$ are all nonnegative definite due to the addition formula (1.5). Being the coefficients $\beta_{l, k}^{m}$ and $\left(1-\lambda^{2}\right)^{l}$ all positive, our assumption on $\lambda$ shows that the previous equality is equivalent to

$$
\sum_{x_{\varepsilon}^{\prime} \in W_{\varepsilon}^{\prime}} \sum_{y_{\varepsilon}^{\prime} \in W_{\varepsilon}^{\prime}} R_{l}^{m-1}\left(x_{\varepsilon}^{\prime} \cdot y_{\varepsilon}^{\prime}\right)=0, \quad l=0,1, \ldots, q .
$$

Let $x^{\prime \prime}$ and $y^{\prime \prime}$ be points of $S^{m-2}$ so that $\Psi_{\varepsilon}\left(x^{\prime \prime}\right)=x_{\varepsilon}^{\prime}$ and $\Psi_{\varepsilon}\left(y^{\prime \prime}\right)=y_{\varepsilon}^{\prime}$. Hence,

$$
\begin{aligned}
0 & =\sum_{x_{\varepsilon}^{\prime} \in W_{\varepsilon}^{\prime}} \sum_{y_{\varepsilon}^{\prime} \in W_{\varepsilon}^{\prime}} R_{l}^{m-1}\left(x_{\varepsilon}^{\prime} \cdot y_{\varepsilon}^{\prime}\right) \\
& =\sum_{x_{\varepsilon}^{\prime} \in W_{\varepsilon}^{\prime}} \sum_{y_{\varepsilon}^{\prime} \in W_{\varepsilon}^{\prime}} R_{l}^{m-1}\left(x^{\prime \prime} \cdot y^{\prime \prime}\right) \\
& =\sum_{x^{\prime \prime} \in \Psi_{\varepsilon}^{-1}\left(W_{\varepsilon}^{\prime}\right)} \sum_{y^{\prime \prime} \in \Psi_{\varepsilon}^{-1}\left(W_{\varepsilon}^{\prime}\right)} R_{l}^{m-1}\left(x^{\prime \prime} \cdot y^{\prime \prime}\right), \quad l=0,1, \ldots, q .
\end{aligned}
$$

Theorem 2.1 once again shows that $\Psi_{\varepsilon}^{-1}\left(W_{\varepsilon}^{\prime}\right)$ is a $q$-design in $S^{m-2}$.

In the last theorem of the paper, we shall deal with a design $W$ of $S^{m-1}$, looking at its rim decomposition with respect to a fixed pole $\varepsilon$ of $S^{m-1}$. Precisely, we shall write $W$ as a disjoint union of the form $W=\dot{\cup}_{i=1}^{n} W_{\lambda_{i}}$, where

$$
W_{\lambda_{i}}=W \cap\left\{x \cdot \varepsilon=\lambda_{i}: x \in S^{m-1}\right\}, \quad \lambda_{i} \in(-1,1), \quad i=1,2, \ldots, n,
$$

and will investigate the effect of rotations on the $W_{\lambda_{i}}$. The result can be seen as an extension of a result proved by Yudin ([12]).

Theorem 3.3. Let $W$ be a finite and nonempty subset of $S^{m-1}$. Consider the rim decomposition $W=\dot{\cup}_{i=1}^{n} W_{\lambda_{i}}$ of $W$ with respect to $\varepsilon$. Let $\rho_{1}, \rho_{2}, \ldots, \rho_{n}$ be elements of $\mathcal{O}_{m}$ so that $\rho_{j}(\varepsilon)=\varepsilon, j=1,2, \ldots, n$. If $W$ is a q-design and every $W_{\lambda_{i}, \varepsilon}^{\prime}$ is a $q_{i}$-design, $i=1,2, \ldots, n$, then $W_{\rho}:=\cup_{i=1}^{n} \rho_{i}\left(W_{\lambda_{i}}\right)$ is a $\nu$-design, where $\nu=$ $q \wedge q_{1} \wedge \ldots \wedge q_{n}$

Proof. Assuming that $W$ is a $q$-design and each $W_{\lambda_{i}, \varepsilon}^{\prime}$ is a $q_{i}$-design, we shall verify that

$$
\sum_{x \in W_{\rho}} R_{n}^{m}(x \cdot y)=0, \quad y \in S^{m-1}, \quad n=1,2, \ldots, \nu .
$$

If every $\rho_{j}$ is the identity operator, then $W_{\rho}=W$. Since $W$ is a $q$-design, (3.8) follows from Theorem 2.1 and the inequality $\nu \leq q$. In the general case, we write

$$
\sum_{x \in W_{\rho}} R_{n}^{m}(x \cdot y)=\sum_{i=1}^{n} \sum_{x \in \rho_{i}\left(W_{\lambda_{i}}\right)} R_{n}^{m}(x \cdot y),
$$

and verify that the right-hand side does not depend upon $\rho_{i}, i=1,2, \ldots, n$, when $n \leq \nu$. To do that, we shall look at each summand separately. Fix $i \in\{1,2, \ldots, n\}$. 
Since each $\rho_{i}$ preserves inner product,

$$
\sum_{x \in \rho_{i}\left(W_{\lambda_{i}}\right)} R_{n}^{m}(x \cdot y)=\sum_{\rho_{i}^{*}(x) \in W_{\lambda_{i}}} R_{n}^{m}\left(\rho_{i}^{*}(x) \cdot \rho_{i}^{*}(y)\right)=\sum_{z \in W_{\lambda_{i}}} R_{n}^{m}\left(z \cdot \rho_{i}^{*}(y)\right) .
$$

Employing the decomposition (3.2) for $z$ and $y$, we deduce that

$$
\begin{aligned}
\sum_{x \in \rho_{i}\left(W_{\lambda_{i}}\right)} R_{n}^{m}(x \cdot y) & =\sum_{z \in W_{\lambda_{i}}} R_{n}^{m}\left(\lambda_{i} \xi+\left(1-\lambda_{i}^{2}\right)^{1 / 2}\left(1-\xi^{2}\right)^{1 / 2}\left(\rho_{i}\left(z_{\varepsilon}^{\prime}\right) \cdot y_{\varepsilon}^{\prime}\right)\right) \\
& =\sum_{z_{\varepsilon}^{\prime} \in W_{\lambda_{i}}^{\prime}} R_{n}^{m}\left(\lambda_{i} \xi+\left(1-\lambda_{i}^{2}\right)^{1 / 2}\left(1-\xi^{2}\right)^{1 / 2}\left(\rho_{i}\left(z_{\varepsilon}^{\prime}\right) \cdot y_{\varepsilon}^{\prime}\right)\right)
\end{aligned}
$$

where $\xi=z \cdot \varepsilon$. The index arrangement in the last sum is justified by the fact that $z \in W_{\lambda_{i}} \rightarrow z_{\varepsilon}^{\prime} \in W_{\lambda_{i}}^{\prime}$ is one-to-one. Since $R_{n}^{m}$ is a polynomial of degree $n$, there are coefficients $a_{l}\left(\lambda_{i}, \xi\right), l=0,1, \ldots, n$, so that

$$
\sum_{x \in \rho_{i}\left(W_{\lambda_{i}}\right)} R_{n}^{m}(x \cdot y)=\sum_{l=0}^{n} a_{l}\left(\lambda_{i}, \xi\right)\left(1-\lambda_{i}^{2}\right)^{l / 2} \sum_{z_{\varepsilon}^{\prime} \in W_{\lambda_{i}}^{\prime}}\left(z_{\varepsilon}^{\prime} \cdot \rho_{i}^{*}\left(y_{\varepsilon}^{\prime}\right)\right)^{l} .
$$

Since $W_{\lambda_{i}}^{\prime}$ is a $q_{i}$-design and $n \leq \nu$, Theorem 2.1-(iii) can be used to deduce that

$$
\begin{aligned}
\sum_{x \in \rho_{i}\left(W_{\lambda_{i}}\right)} R_{n}^{m}(x \cdot y) & =\left|W_{\lambda_{i}}^{\prime}\right| \sum_{\substack{l=0 \\
l \text { par }}}^{n} a_{l}\left(\lambda_{i}, \xi\right)\left(1-\lambda_{i}^{2}\right)^{l / 2}\left(\frac{1}{\left|W_{\lambda_{i}}^{\prime}\right|} \sum_{z_{\varepsilon}^{\prime} \in W_{\lambda_{i}, \varepsilon}^{\prime}}\left(z_{\varepsilon}^{\prime} \cdot \rho_{i}^{*}\left(y_{\varepsilon}^{\prime}\right)\right)^{l}\right) \\
& =\left|W_{\lambda_{i}}^{\prime}\right| \sum_{\substack{l=0 \\
l \text { par }}}^{n} a_{l}\left(\lambda_{i}, \xi\right)\left(1-\lambda_{i}^{2}\right)^{l / 2} \alpha_{l}\left\|\rho_{i}^{*}\left(y_{\varepsilon}^{\prime}\right)\right\|^{l}
\end{aligned}
$$

It follows that

$$
\sum_{x \in \rho_{i}\left(W_{\lambda_{i}}\right)} R_{n}^{m}(x \cdot y)=\left|W_{\lambda_{i}}^{\prime}\right| \sum_{\substack{l=0 \\ l \text { par }}}^{n} a_{l}\left(\lambda_{i}, \xi\right)\left(1-\lambda_{i}^{2}\right)^{l / 2} \alpha_{l}\left\|y_{\varepsilon}^{\prime}\right\|^{l}
$$

which is an expression not depending on $\rho_{i}$. Therefore, the theorem follows from the considerations at the beginning of the proof and Theorem 2.1-(ii).

At least two consequences of the Theorem 3.3. are easily obtained.

Corollary 3.4. Let $W$ be a finite nonempty subset of $S^{m-1}, \varepsilon \in S^{m-1}$ and $\lambda \in$ $(-1,1)$. Let $\rho \in \mathcal{O}_{m}$ so that $\rho(\varepsilon)=\varepsilon$. If $W$ is a $q$-design and $W_{\lambda}^{\prime}$ is a $q_{1}$-design, then $W_{\rho}=\left(W \backslash W_{\lambda}\right) \cup \rho\left(W_{\lambda}\right)$ is a $q \wedge q_{1}$-design.

Proof. It suffices to adapt the proof of Theorem 3.3.

In Corollary 3.5, we shall specialize the pole $\varepsilon$. We shall assume that one of its components equals 1 . We shall write $W_{\lambda}^{\prime \prime}$ to denote the subset of $S^{m-2}$ obtained 
from $W_{\lambda}^{\prime}$, when we ignore the zero components of its elements which correspond to the nonzero entry of $\varepsilon$.

Corollary 3.5. Let $W$ be a finite nonempty subset of $S^{m-1}, \lambda \in(-1,1)$ e $\rho \in \mathcal{O}_{m}$. Let $\varepsilon$ be as in the previous paragraph. If $W$ is a q-design and $W_{\lambda}^{\prime \prime}$ is a $q_{1}$-design in $S^{m-2}$, then $W_{\rho}=\left(W \backslash W_{\lambda}\right) \cup \rho\left(W_{\lambda}\right)$ is a $q \wedge q_{1}$-design.

Proof. It will be omitted.

Let $W_{2}$ be the set considered in the previous section and $\varepsilon=(0,0,1) \in \mathbb{R}^{3}$. The rotation $\rho \in \mathcal{O}_{3}$ defined by

$$
\rho(x, y, z)=\left(\begin{array}{ccc}
\cos \theta & \sin \theta & 0 \\
-\sin \theta & \cos \theta & 0 \\
0 & 0 & 1
\end{array}\right)\left(\begin{array}{l}
x \\
y \\
z
\end{array}\right)
$$

satisfies $\rho(\varepsilon)=\varepsilon$. If $\lambda=0$ then $W_{\lambda}=W_{\lambda}^{\prime}=\{(1,0,0),(0,1,0),(-1,0,0),(0,-1,0)\}$ and $W_{\lambda}^{\prime \prime}=\{(1,0),(0,1),(-1,0),(0,-1)\}$. Since $W_{\lambda}^{\prime \prime}$ coincides with the design $W_{1}$ analyzed in the previous section, $W_{\lambda}^{\prime \prime}$ is a 3 -design in $S^{1}$. A procedure similar to that used in Section 2 shows that $W$ is a 3-design in $S^{2}$. Hence, Corollary 3.5 is applicable and in the case $\theta=\pi / 4$ we can conclude that the union of

$$
W \backslash W_{\lambda}=\{(0,0,1),(0,0,-1)\}
$$

and

$$
\rho\left(W_{\lambda}\right)=\left\{\left(\frac{\sqrt{2}}{2},-\frac{\sqrt{2}}{2}, 0\right),\left(\frac{\sqrt{2}}{2}, \frac{\sqrt{2}}{2}, 0\right),\left(-\frac{\sqrt{2}}{2}, \frac{\sqrt{2}}{2}, 0\right),\left(-\frac{\sqrt{2}}{2},-\frac{\sqrt{2}}{2}, 0\right)\right\}
$$

is a 3 -design in $S^{2}$.

\section{References}

[1] R. Askey, "Orthogonal Polynomials and Special Functions", Philadelphia: Society for Industrial and Applied Mathematics, 1975, Regional Conference Series in Applied Mathematics, 21, 1974.

[2] S. Axler, P. Bourdon, W. Ramey, "Harmonic Function Theory", Graduate Texts in Mathematics, 137. Springer-Verlag, New York, 1992.

[3] L. Carlitz, The product of two ultraspherical polynomials, Proc. Glasgow Math. Assoc. 5 (1961), 76-79.

[4] P. Delsarte, J.M. Goethals, J.J. Seidel, Spherical codes and designs, Geometriae Dedicata, 6, No. 3 (1977), 363-388.

[5] J.M. Goethals, J.J., Seidel, Spherical designs. Relations between combinatorics and other parts of mathematics (Proc. Sympos. Pure Math., Ohio State Univ., Columbus, Ohio, 1978), pp. 255-272, Proc. Sympos. Pure Math., XXXIV, Amer. Math. Soc., Providence, R.I., 1979. 
[6] J.M. Goethals, J.J. Seidel, The football, Nieuw Arch. Wisk. (3) 29, No. 1 (1981), 50-58.

[7] H. Groemer, "Geometric Applications of Fourier Series and Spherical Harmonics". Encyclopedia of Mathematics and its Applications, vol. 61, Cambridge University Press, Cambridge, 1996.

[8] E. Hylleraas, Linearization of products of Jacobi polinomials, Math. Scand., 10 (1962), 189-200.

[9] C. Müller, "Analysis of Spherical Symmetries in Euclidean Spaces", Applied Mathematical Sciences, 129. Springer-Verlag, New York, 1998.

[10] E.M. Stein, G. Weiss, "Introduction to Fourier Analysis on Euclidean Spaces", Princeton Mathematical Series, No. 32. Princeton University Press, Princeton, N.J., 1971.

[11] G. Szego", "Orthogonal Polynomials", American Mathematical Society Colloquium Publications, v. 23. American Mathematical Society, New York, 1939.

[12] V.A. Yudin, Rotation of spherical designs. (Russian) Problemy Peredachi Informatsii 36, No. 3 (2000), 39-45; translation in Probl. Inf. Transm. 36, No. 3 (2000), 230-236. 